\title{
Percepção da equipe multiprofissional: um cuidado necessário a pessoa com obesidade na unidade de terapia intensiva
}

\author{
Perception of the multiprofessional team: a \\ necessary care for persons with obesity in \\ the intensive care unit
}

\author{
Luciara Fabiane Sebold ${ }^{1}$ (C) \\ Juliana Balbinot Reis Girondi ${ }^{2}$ (]) \\ Lúcia Nazareth Amante ${ }^{3}$
}

\author{
Bruna Machado Pardal 4 \\ Thainá de Souza Kagaochi ${ }^{5}$ \\ Bárbara Mohr da Silveira ${ }^{6}$ (1)
}

${ }^{1}$ Autora para correspondência. Universidade Federal de Santa Catarina (Florianópolis). Santa Catarina, Brasil. fabisebold@gmail.com 2-6Universidade Federal de Santa Catarina (Florianópolis). Santa Catarina, Brasil. juliana.balbinot@ufsc.br, luciamante@gmail.com, brunapardal@gmail.com, tkagaochi@gmail.com, barbaramohrs@gmail.com

RESUMO | OBJETIVO: Conhecer a percepção da equipe multiprofissional sobre o cuidado à pessoa com obesidade em uma Unidade de Terapia Intensiva (UTI). MÉTODO: Estudo exploratório e descritivo, com abordagem qualitativa, desenvolvido em uma UTI do sul do Brasil, com 14 profissionais da equipe de saúde. Os dados foram coletados por meio de entrevista semiestruturada, entre junho e setembro de 2018, e a análise se deu por meio da análise de conteúdo de Bardin. RESULTADOS: Após análise das informações coletadas dos relatos dos participantes, emergiram quatro categorias: Dificuldades para a realização do cuidado; Cuidados Específicos; Gestão do cuidado: Equipe de Trabalho; Sentimentos e reações diante do cuidado às pessoas com obesidade. A equipe de saúde percebe algumas dificuldades para a realização do cuidado, mas que alguns são especiais, a gestão do cuidado está pautada na equipe de trabalho e no contexto ético. CONCLUSÕES: Os cuidados prestados pela equipe de saúde à pessoa com obesidade são realizados mesmo diante das adversidades relacionadas aos materiais, equipamentos, número de profissionais e questões éticas.

DESCRITORES: Obesidade. Unidade de Terapia Intensiva. Equipe de Assistência ao Paciente. Percepção.

\begin{abstract}
OBJECTIVE: To know the perception of the multidisciplinary team about the care of people with obesity in an Intensive Care Unit (ICU). METHOD: Exploratory and descriptive study, with a qualitative approach, developed in an ICU in southern Brazil, with 14 professionals from the health team. Data were collected through semi-structured interviews between June and September 2018, and the analysis took place through Bardin's content analysis. RESULTS: After analyzing the information collected from the participants' reports, four categories emerged: Difficulties in providing care; Specific Care; Care Management: Work Team; Feelings and reactions towards caring for people with obesity. The health team perceives some difficulties in providing care, but that some are special, care management is based on the work team and the ethical context. CONCLUSIONS: The care provided by the health team to people with obesity is carried out even in the face of adversities related to materials, equipment, number of professionals, and ethical issues.
\end{abstract}

DESCRIPTORS: Obesity. Intensive Care Units. Patient Care Team. Perception. 


\section{Introdução}

A obesidade é o excesso de peso corporal na forma de gordura, e quando acumulada, pode levar a graves problemas de saúde. E é considerada um dos mais importantes problemas de saúde pública no mundo. ${ }^{1}$

Existem vários meios para medir e identificar a obesidade, o mais usado é o Índice de Massa Corporal (IMC). O excesso de peso inclui o sobrepeso e a obesidade, sendo o primeiro definido como IMC entre 25$29,9 \mathrm{~kg} / \mathrm{m}^{2}$, e o segundo, como IMC $\geq 30 \mathrm{~kg} / \mathrm{m}^{2}$, que define obesidade. 1 A obesidade ainda é dividida em graus I, II e III, sendo considerada obesidade grau I, com IMC entre $30-34,9 \mathrm{~kg} / \mathrm{m}^{2}$, grau II, com IMC de 30 a $39,9 \mathrm{~kg} / \mathrm{m}^{2}$, e grau III, aquele com IMC maior que $\geq 40,0 \mathrm{~kg} / \mathrm{m}^{2}$.

De acordo com a Organização Mundial da Saúde², a prevalência mundial da obesidade quase triplicou entre 1975 e 2016, e existem cerca de dois bilhões de adultos com excesso de peso, dos quais, 650 milhões são considerados obesos. Isso equivale a $39 \%$ de adultos com 18 anos ou mais acima do peso e $13 \%$ de obesos. Estima-se que a maioria da população mundial vive em países onde há excesso de peso.

No Brasil, o excesso de peso da população cresceu, passando de 42,6\% em 2006 para 55,4\% em 2019. A cidade de Manaus (AM) tem a maior prevalência de excesso de peso, e Vitória (ES), a menor. A obesidade cresceu $46 \%$ em dez anos, chegando a $20,3 \%$ em 2019, com frequência semelhante entre os sexos. Dados apontam que em 2019 eram 20,3\% de obesos no Brasil. $\underline{\underline{3}}$

O crescimento da prevalência da obesidade coaduna com o aumento de outras doenças crônicas, como hipertensão arterial sistêmica, diabetes mellitus, entre outras, é provável que, em vários momentos, devido a comorbidades ou por outras necessidades de saúde, pessoas obesas precisem ser internadas em uma UTI. ${ }^{4}$ A obesidade está associada a várias comorbidades, alterações fisiológicas, limitações físicas e alterações farmacocinéticas que podem interferir na evolução da doença aguda e impedir e/ou prejudicar a implementação ou eficácia de intervenções nas UTIs. 5

Para atender às necessidades da pessoa internada na Unidade de Terapia Intensiva (UTI) com segurança e qualidade, é necessário observar entre os vários aspectos relacionados, os recursos terapêuticos disponíveis, a competência e o dimensionamento da equipe multidisciplinar. ${ }^{4}$

Diante das colocações acima, o objetivo do estudo foi conhecer a percepção dos profissionais da saúde sobre o cuidado realizado com a pessoa obesa em UTI.

\section{Método}

Trata-se de um estudo exploratório e descritivo, com abordagem qualitativa, realizado em uma Unidade de Terapia Intensiva de um hospital universitário do sul do Brasil.

Participaram do estudo 14 profissionais de saúde, selecionados por conveniência, sendo seis técnicos de enfermagem, três enfermeiros, uma fonoaudióloga, uma médica, dois fisioterapeutas e uma psicóloga. O critério de inclusão foi: profissionais com no mínimo um ano de atuação na UTI estudada. E de exclusão: profissionais que não prestavam cuidados diretos aos pacientes e profissionais afastados de suas atividades laborais.

A seleção da amostra foi por conveniência, de acordo com a intenção da pesquisadora. .6 Os dados foram coletados através de entrevistas semiestruturadas, que abordavam as percepções de cuidado à pessoa obesa e as tecnologias utilizadas. O período da coleta foi entre junho e setembro de 2018. Os participantes foram abordados de maneira presencial durante o período de trabalho. As entrevistas foram realizadas em uma sala privativa na UTI, foram gravadas em aparelho digital de voz e, posteriormente, transcritas na íntegra, com duração de cerca de 30 minutos.

A análise dos dados se deu por meio da análise de conteúdo de Bardin, que visa categorizá-los para meIhor interpretação dos achados. A análise de conteúdo pode ser compreendida em três fases: pré-análise, exploração do material e tratamento dos resultados.?

Este estudo faz parte de um macroprojeto intitulado Redes de Atenção à Saúde: Tecnologias de Cuidado à Pessoa com Sobrepeso elou obesidade na Grande Florianópolis/Santa Catarina, respeitando todos os preceitos éticos. Foi aprovado pelo Comitê de Ética em Pesquisa, número do parecer: 1.631.404. Os sujeitos assinaram o Termo de Consentimento Livre e Esclarecido (TCLE). 
Para manter o anonimato dos participantes do estudo, os mesmos foram intitulados com a letra inicial de cada profissão, seguida pelo número ordinal correspondente à ordem de realização da pesquisa.

\section{Resultados}

Após análise das informações coletadas dos relatos dos participantes, emergiram quatro categorias: Dificuldades para a realização do cuidado; Cuidados Específicos; Gestão do cuidado: Equipe de Trabalho; Sentimentos e reações diante do cuidado às pessoas com obesidade.

\section{Dificuldades para a realização do Cuidado}

A dificuldade de prestar um cuidado adequado e eficiente ficou evidenciada nos depoimentos dos participantes do estudo quanto à falta de materiais e equipamentos na UTI estudada. Segundo a maioria dos participantes do estudo, os materiais não são apropriados para o tamanho das pessoas, destacando-se os seguintes relatos:

Seria bom ter uma cama para pessoa obesa, bota pneumática... Fraldas maiores, aqui se usam duas fraldas juntas e abertas (E1).

Acho que uma cama apropriada, pelo menos uma cama para obeso deveria ter, porque essa nossa cama é assim, se for um obeso mórbido, a gente não consegue nem levantar e ela emperra (T5).

A cama é pequena, dificulta a mudança de decúbito. $O$ colchão de ar tem limite de peso, ele não liga. A própria

cama, para levantar a cabeceira às vezes trava. $O$ manguito é pequeno, na verdade a gente tem ele largo, mas é pequeno... e as gordinhas baixinhas tem o braço pequeno, aí dificulta (T2).

As fraldas são pequenas então não dá para fechar. Geralmente a gente deixa aberta e coloca mais de uma.

Ou então cola uma na outra e deixa aberta, porque não tem condições de fechar, elas são pequenas mesmo (T1).

A falta de tecnologias adequadas ao manejo das pessoas com obesidade surgiu como uma preocupação:
Sim, tecnologias facilitariam o cuidado, o manejo e até a segurança do paciente obeso. Como por exemplo, o eleve, aparelhos de mobilização passiva, como o cicloergonômetro passivo, colchões específicos, camas específicas... assim como aparatos para facilitar a mobilização e manuseio, como pranchas ortostáticas (para sentar, específicos para eles né. Aparelhos que facilitariam o ortotatismo e sedestação também seria interessante (F1).

Tudo é mais difícil. Sinto dificuldade maior em puncionar PAM, Cateter Central, realizar intubação... conseguimos após várias tentativas e muitos materiais gasto (M1).

As tecnologias para o cuidado podem ser um grande diferencial na recuperação da pessoa com obesidade. Por conseguinte, um ambiente complexo como o de uma Unidade de Terapia Intensiva pode ser adaptado para tal objetivo.

\section{Cuidados Específicos}

Os cuidados com a pele das pessoas com obesidade foram relatos frequentes dos participantes do estudo. Foram salientados temas que retratam os cuidados como banho no leito, monitorização, reposicionamento e posicionamento.

A utilização de cremes e pomadas específicas para evitar lesão por pressão foram evidentes, demonstrando sua importância:

O cuidado com a pele mesmo, eu passo em todas as dobrinhas creme para assaduras, porque sempre fica molhado e abre logo (T2).

Presto cuidado no banho, tem que secar bem o paciente para não ficar molhada a pele e não assar (T3).

Aplicação de cremes, pomada vitaminada, em áreas que acontecem cisalhamento, posicionar coxins em áreas de proeminência (E3).

Também foram lembradas ações quanto ao posicionamento e reposicionamento, e os obstáculos enfrentados pela equipe:

Uso coxins aqui, aqui e outro aqui para melhor posicionar o paciente. No banho tem que secar bem as dobras e passar pomada vitaminada para evitar assaduras (T5) 
"...é um paciente que tem tudo para abrir lesão, porque tu não vai fazer todas as mudanças de decúbito. Tipo, as vezes não dá para virar o paciente de lado, é bem complicado... a gente já pegou paciente que tomava conta da cama inteira, ai é bem difícil fazer mudança (T5).

Uma boa monitorização... observar saturação, frequência cardíaca, o ventilador, pois uma mudança pode alterar a hemodinâmica do paciente (M1).

As técnicas adequadas de mobilização e movimentação previnem complicações indesejadas. Atentar para o risco de potenciais lesões e avaliar a hemodinâmica previamente são precauções essenciais para a saúde da pessoa com obesidade na UTI.

\section{Gestão do cuidado: Equipe de trabalho}

Nesta categoria, foram agrupadas estratégias utilizadas para minimizar a sobrecarga do trabalho e tentar otimizar o cuidado à pessoa com obesidade, que estão representadas nos depoimentos abaixo:

"... a gente mexe em todos os outros e quando está todo mundo livre, a gente vai todo mundo junto no mesmo paciente. Esse é o mais certo. Ou vai todo mundo antes, que é bem raro" (T2).

Não consigo colocar eles para cima sozinha, eu procuro pedir ajuda pra equipe de enfermagem, geralmente para o técnico que está com ele e também procuro trabalhar com o paciente quando o técnico já fez uma parte do seu trabalho (FO1).

“...às vezes precisa de 4 pessoas para passar uma sonda. Cada uma numa perna, uma na barriga. Quando tu te dá conta, tem seis funcionários em volta do paciente" (E1).

Para mobilizar eu procuro pedir ajuda dos colegas para não machucar o paciente e também a gente. Às vezes duas pessoas são impossíveis para mobilizar um paciente e nem sempre têm pessoas disponíveis, às vezes ela está ocupada com outras coisas e daí você acaba se obrigando a virar o paciente sozinho, e com o passar dos anos acaba acarretando prejuízo para nossa saúde... e às vezes até machuca o paciente (T5).

Os sentimentos trazidos pela equipe em relação às dificuldades encontradas no cuidado foram, de certa forma, voltados para a cooperação na realização das atividades cotidianas com a pessoa com obesidade.
Sempre tem uma dificuldade maior. Dificuldade para movimentar o paciente, porque depende dos colegas, a gente necessita de ajuda deles. É preciso um número maior de pessoas para mobilizar o paciente porque não consigo fazer sozinha (T6).

Tenho dificuldade de realizar mobilização articular passiva, manobras de higiene brônquica, colocar sozinha os pacientes sentados e em pé (F2).

A equipe intensivista reconhece a união e trabalha em conjunto dedicando-se a um objetivo comum: o cuidado adequado aos seus pacientes.

\section{Sentimentos e reações diante do cuidado às pessoas com obesidade}

Verificou-se, por meio dos depoimentos, que alguns profissionais durante o cuidado à pessoa com obesidade apresentam dificuldade de se colocar no lugar do outro e emitem juízos de valores e expressam em seus depoimentos sentimentos de indignação em relação às manifestações de outros profissionais.

"Já ouvi colegas se referindo ao paciente chamando ele de 'gordinho' ao invés pelo nome ou leito..." (M1).

"Têm pessoas que não tem noção. Fazem muitos comentários a respeito do paciente. Não deveriam nem pensar, quanto mais falar. Eu fico abismada nas passagens de plantão com alguns tipos de comentários" (P1).

"Uma vez escutei uma médica falar: está aí ele, estou trazendo mais um gordinho para vocês" (F1).

Eu vejo bastante, de toda a equipe multiprofissional sabe, às vezes eu fico constrangida com alguns comentários que surgem a respeito daquele paciente obeso... o paciente tá ali, lúcido, acordado e as pessoas não tem filtro né. Deveria entrar como um protocolo... não fazer piada, não fazer comentários do tipo 'Meu Deus! Como que a pessoa chegou nesse tamanho?' É

lógico que a gente fala, todo mundo fala, mas ter o cuidado de não falar isso na frente da pessoa, pra ela não escutar, porque imagina você lá, impotente nessa cama, ouvindo este tipo de comentário (T5).

Atenta-se também aos depoimentos dos participantes do estudo quando questionados sobre a sensação de chegar no trabalho e ver uma pessoa com obesidade internada na UTI: 
Quando eu vejo um obeso na UTI chega a me dar um

desânimo (T2).

“... nossa, eu não posso falar isso, mas eles são muito grandes e me cansam muito" (T3).

A equipe multiprofissional é responsável pela qualidade da assistência. Deve-se promover a humanização das UTIs com o objetivo de valorizar a essência da pessoa, atribuindo respeito, dignidade, cooperação e conformação.

\section{Discussão}

Os participantes revelaram as dificuldades na realização do cuidado por falta de tecnologia apropriada para pessoas obesas, evidenciado, também, em um estudo goiano ${ }^{8}$, no qual $70 \%$ dos entrevistados afirmaram que $\mathrm{o}$ ato de cuidar de uma pessoa com obesidade é muito difícil, e $85 \%$ que a falta de material adequado interfere no serviço prestado. Uma pesquisa no interior do sul do Brasil ${ }^{9}$ identificou que a precariedade ou a falta de equipamentos e materiais também são considerados obstáculos, pois a ausência de infraestrutura adequada compromete o processo de cuidar, especialmente em uma UTI. Na atual pesquisa, observou-se que há dificuldade para prestar assistência adequada devido à falta de materiais e equipamentos na UTI, como camas apropriadas para pessoas com obesidade, manguitos e fraldas maiores.

A legislação brasileira, por meio da Portaria $n^{\circ} 390$ de 06 de julho de $2005^{10}$, institui diretrizes para a Atenção ao Paciente Portador de Obesidade, e traz que a Unidade deverá dispor de todos os materiais e equipamentos necessários em perfeito estado de conservação e funcionamento, para assegurar a qualidade da assistência às pessoas com obesidade. Tais materiais incluem: cama hospitalar do tipo Fowler especial para pessoas com obesidade com capacidade para $350 \mathrm{~kg}$; poltrona reclinável específica para paciente com mais de $300 \mathrm{Kg}$; maca para transporte e cadeira de rodas que aguentem até $350 \mathrm{Kg}$; roupas específicas para pacientes acima de $200 \mathrm{~kg}$, tais como camisolas, roupões e pijamas; respiradores volumétricos que suportam volumes e pressões especialmente elevadas nos pacientes superobesos; manguitos e esfigmomanômetros especiais.
A gama tecnológica se renova e cresce. tornando os equipamentos mais rápidos e precisos em suas funções aperfeiçoando o processo de cuidar. Nesse sentido, podemos perceber que o avanço pode repercutir diretamente na assistência prestada ao paciente crítico, influenciando nos padrões de diagnósticos e tratamentos. ${ }^{11}$

O cuidado centrado no paciente, através da humanização, o holismo e a segurança do paciente, são características aplicadas na prática das atividades desenvolvidas na rotina da UTI, pois estas garantem uma assistência de qualidade. ${ }^{\frac{12}{}} \mathrm{E}$, assim, os cuidados interdisciplinares tendem a produzir maior perda de peso, clinicamente significativa e sustentada, em comparação aos cuidados habituais, bem como meIhora em outros desfechos em saúde, demonstrando que a prevenção e controle da obesidade torna-se mais eficaz nesta modalidade de atenção à saúde. $\cdot^{13}$

A atuação do fisioterapeuta na UTI consiste no auxílio do suporte ventilatório, onde o mesmo é capacitado e detentor de conhecimentos específicos, podendo atuar em todas as etapas, e que, por sua vez, faz parte do plano terapêutico que assiste o paciente, visando à melhora de seu quadro clínico..$^{14} \mathrm{O}$ fisioterapeuta intensivista é o profissional responsável pelo diagnóstico fisioterapêutico por meio da avaliação físico-funcional, reduzindo a chance de possíveis complicações clínicas e infecções e trazendo muitos benefícios aos pacientes graves, em razão de maior vulnerabilidade e complicações súbitas. $\frac{15}{}$

Desta forma, a fisioterapia respiratória possui um papel importante no pós-operatório, atuando em forma de prevenção e tratamento nas possíveis complicações que possam vir a acontecer ou que já estejam instalados no paciente. A fim de melhorar, tratar e prevenir esses pacientes que passam por essa intervenção cirúrgica são utilizadas técnicas de higiene brônquica, expansão pulmonar, treinamento respiratório, uso de ventilação não invasiva e invasiva, CPAP, BiPAP, lembrando que cada paciente possui um diagnóstico. No entanto, cada tratamento será realizado de acordo com a necessidade de cada paciente. ${ }^{16} \mathrm{Na}$ atual pesquisa, ao observar os relatos dos fisioterapeutas, nota-se que eles realizam mobilização e exercícios com os pacientes, porém se deparam com dificuldades. 
Outro aspecto observado no presente estudo foram as dificuldades de posicionamento e reposicionamento do paciente. Esta não mobilização pode contribuir para o aparecimento de lesões, já que representa cuidados diários com a pele que são de suma importância. Considera-se que a caracterização do paciente hospitalizado com lesões de pele contribui para planejar e implantar assistência integral e sistematizada. Além disso, pode subsidiar a atualização de protocolos clínicos específicos de prevenção e tratamento das lesões de pele, em especial a LP. ${ }^{17}$

Uma forma de avaliar o risco de lesão por pressão é por meio da utilização da Escala de Braden, permitindo que o enfermeiro possa mensurar de forma clara e objetiva o risco de cada paciente para o desenvolvimento da LP, além de fornecer subsídios para a elaboração dos diagnósticos de enfermagem que são responsáveis por reduzir a incidência da lesão. Ela é composta por seis subescalas que constituem a percepção sensorial, a umidade da pele, o estado nutricional, o grau de atividade e mobilidade, a exposição à fricção e cisalhamento. A somatória dos escores resulta em valores entre 6 e 23; quanto menor a pontuação, maior o risco para ocorrer a lesão. ${ }^{18}$

No estudo norte-americano ${ }^{\frac{19}{}}$, três tipos de camas foram utilizadas nas UTIs: regular, especial e bariátrica. Entre os pacientes da amostra, $89,5 \%$ usaram a cama regular, 4,1\% utilizaram a cama especial e $6,4 \%$ utilizaram a cama bariátrica. A incidência de úlcera por pressão foi $34 \%$ para pacientes colocados na cama especial, $25 \%$ para a cama bariátrica e $5 \%$ para a cama normal. No mesmo estudo, concluiu-se que os pacientes extremamente obesos tinham cerca de duas vezes mais probabilidade de sofrer lesão do que os pacientes com peso normal.

Um dos aspectos enfatizados pelos participantes deste estudo foi a questão da gestão do cuidado, especificamente para a equipe de trabalho, que demonstra que o cuidado à pessoa obesa gera, na equipe de saúde, uma demanda de esforço físico e divisão de tarefas. Destaca-se então que a valorização do cuidado à pessoa obesa necessita estar associada à qualificação dos profissionais que cuidam dessa clientela, uma vez que o cuidado não se faz somente por estruturas, mas fundamentalmente por pessoas; entre as quais, os profissionais que nela atuam assumem papel fundamental para uma efetiva gestão do cuidado à obesidade
Um estudo paulista ${ }^{4}$ concluiu que a carga de trabalho vivenciada pela equipe de enfermagem, na UTI, segundo o Nursing Activities Score (NAS), não difere entre os grupos com diferentes IMC. Entretanto, destaca-se que o quantitativo insuficiente de profissionais para a realização de cuidados que envolvem mobilização da pessoa com obesidade com dependência total, pode contribuir para maior risco na ocorrência de eventos adversos, além de gerar sobrecarga física nos profissionais. As pessoas com obesidade demandam mais tempo para o procedimento de higienização e maior número de pessoas para mobilização/posicionamento. Quanto aos depoimentos referentes à carga de trabalho prestada na UTI do hospital estudado, percebe-se que os profissionais não conseguem trabaIhar sozinhos, tampouco com a ajuda de apenas um colega, necessitando de mais colegas para movimentar um paciente obeso, seja nas trocas de fraldas, banho de leito ou mobilização.

Quanto à ética dos profissionais de saúde no cuidado à pessoa obesa, encontraram-se poucos achados científicos. Em uma revisão integrativa ${ }^{20}$, evidenciouse que avaliações preconceituosas estão presentes nas diversas profissões da área da saúde, prejudicando a assistência, fazendo com que o paciente se sinta excluído e marginalizado. Isso acarreta um afastamento dos sistemas de saúde e propicia um menor cuidado preventivo.

Os aspectos positivos do cuidado mostram a importância do estabelecimento de vínculo entre o profissional de saúde e o cliente, no processo de enfrentamento e superação dos desafios durante a internação, tendo como consequência a valorização do profissional de saúde. Já aspectos negativos do cuidado relatados contradizem a essência da palavra "cuidado", o que demanda reflexões quanto à assistência prestada e à necessidade de se investir cada vez mais em práticas humanizadoras. 11

Neste estudo, observou-se que os profissionais da equipe multiprofissional se empenham com esmero e tentam realizar o cuidado da melhor forma possível, mesmo, às vezes, se deparando com questões desafiadoras em um ambiente complexo como o da UTI. Sendo assim, a valorização das relações humanas, através de intervenções comprometidas com o cuidado integral, baseadas no acolhimento, escuta, comunicação, vínculo e responsabilização fazem com que a equipe multiprofissional preste o melhor cuidado possível.22 


\section{Considerações finais}

A presente pesquisa buscou conhecer as percepções dos profissionais da saúde sobre o cuidado realizado com pessoas com obesidade em terapia intensiva, apontando que a equipe presta o cuidado mesmo diante das adversidades relacionadas aos materiais/ equipamentos. Também tentam garantir os cuidados com a pele por meio da mobilização, utensílios e soluções para prevenção de lesões. Quanto à gestão do cuidado voltado à divisão da equipe de trabalho, os profissionais fazem uso de várias estratégias, como trabalhar em conjunto. Outro aspecto revelado foram as questões éticas, ao serem observados comentários inadequados, algo ofensivo e desnecessário. Apesar de todos os obstáculos, os profissionais devem garantir os direitos a todas as pessoas com obesidade e não os considerar como sujeitos limitantes.

A limitação encontrada no estudo foi em relação ao número reduzido da amostra e o fato do mesmo ter sido realizado em apenas uma instituição hospitalar. Recomenda-se o desenvolvimento de estudos sobre o cuidado à pessoa com obesidade em unidades de terapia intensiva e formas de melhorar a assistência.

Por fim, acentua-se a importância dos cuidados prestados pela equipe multiprofissional à pessoa com obesidade e como as tecnologias são indispensáveis no ambiente hospitalar.

\section{Contribuições das autoras}

Sebold LF e Pardal BM participaram da concepção, delineamento, busca e análise estatística dos dados da pesquisa, interpretação dos resultados e redação do artigo científico. Girondi JBR e Amante LN participaram da redação do artigo científico.

\section{Conflito de interesses}

Nenhum conflito financeiro, legal ou político envolvendo terceiros (governo, empresas e fundações privadas, etc.) foi declarado para nenhum aspecto do trabalho submetido (incluindo, mas não se limitando a subvenções e financiamentos, participação em conselho consultivo, desenho de estudo, preparação de manuscrito, análise estatística, etc.).

\section{Referências}

1. World Obesity Federation. About Obesity [Internet]. Inglaterra: World Obesity; 2018. [citado em 2020 ago. 11]. Disponível em: https://www.worldobesity.org/about/about-obesity

2. World Health Organization. Obesity: Preventing And Managing The Global Epidemic: report of a WHO consultation [Internet]. Geneva: WHO; 2000. [citado em 2020 ago. 11]. Disponível em: http://apps.who.int/iris/bitstream/10665/42330/1/WHO TRS 894. pdf?ua $=1 \& u a=1$

3. Ministério da Saúde (Brasil), Secretaria de Vigilância em Saúde, Departamente do Análise em Saúde e Vigilância de Doenças não transmissíveis. Vigitel Brasil 2019: Vigilância de Fatores de Risco e Proteção para Doenças Crônicas por Inquérito Telefônico [Internet]. Brasília: Ministério da Saúde; 2020. [citado em 2020 ago. 11]. Disponível em: http://www.crn1.org.br/wp-content/ uploads/2020/04/vigitel-brasil-2019-vigilancia-fatores-risco. pdf? $\times 53725$

4. Goulart LL, Sousa FCA, Sueko VZS, Yamaguchi IW. Carga de trabalho de enfermagem relacionada ao índice de massa corporal de pacientes críticos. Acta Paul Enferm. 2017;30(1):31-8. https:// doi.org/10.1590/1982-0194201700006

5. Patel JJ, Rosenthal MD, Miller KR, Codner P, Kiraly L, Martindale RG. The Critical Care Obesity Paradox and Implications for Nutrition Support. Curr. Gastroenterol. Rep. 2016;18:45. https:// doi.org/10.1007/s11894-016-0519-8

6. Polit DF, Beck CT. Fundamentos de Pesquisa em Enfermagem: Avaliação de evidências para a prática da enfermagem. $7^{\text {a }}$ ed. Porto Alegre: Artmed Editora.

7. Bardin L. Análise de conteúdo. Edições 70; 2011.

8. Meireles AB, Lima TO, Jesus ALS, Andrade EGS. Dificuldades no cuidado ao paciente obeso. Rev Inic Cient e Ext. [Internet]. 2018;1(4):328-33. Disponível em: https://revistasfacesa.senaaires. com.br/index.php/iniciacao-cientifica/article/view/113/75

9. Rodrigues IL, Camponogara S, Soares SGA, Beck CLC, Santos TM. Facilidades e dificuldades do trabalho em terapia intensiva: um olhar da equipe de enfermagem. R. pesq. cuid. fundam. online. 2016;8(3):4757-65. https://doi.org/10.9789/21755361.2016.v8i3.4757-4765

10. Portaria SAS/MS № 390, de 06 de julho de 2005 (Brasil). Institui diretrizes para a Atenção ao Paciente Portador de Obesidade. [Internet]. Disponível em: https://www.saude.mg.gov.br/images/ documentos/portaria 0390.pdf

11. Siqueira VRB, Vasconcelos WPC, Santos GM, Oliveira GKS. Contribuições da tecnologia para assistência de enfermagem da unidade de terapia intensiva. Revista Sustinere. 2019;7(1):19-31. https://doi.org/10.12957/sustinere.2019.40086 
12. Pereira MCC, Castro SFF, Brito ES, Carvalho NV, Lopes DV, Pinheiro, JD, et al. Saberes e práticas do enfermeiro na unidade de terapia intensiva. Revi Enferm UFPE on line. 2019;13(1):70-8. https://doi.org/10.5205/1981-8963-v13i1a234842p70-78-2019

13. Tapsell LC, Lonergan M, Batterham MJ, Neale EP, Martin A, Thorne $R$, et al. Effect of interdisciplinary care on weight loss: a randomised controlled trial. BMJ Open. 2017;7(7):e014533. http:// dx.doi.org/10.1136/bmjopen-2016-014533

14. Alves LCBE, Thommen LP, Gomes CM, Oliveira VRC. Conhecimento de acadêmicos da saúde sobre a atuação do fisioterapeuta em unidades de terapia intensiva. RBMC. 2019;5(13). https://doi.org/10.36414/rbmc.v5i13.17

15. Mazzoni AAS. Caracterização das intervenções de Fisioterapia em Unidade de Terapia Intensiva adulto: uma proposta de classificação [dissertação]. Ribeirão Preto: Universidade de São Paulo; 2019. https://doi.org/10.11606/D.22.2019.tde-22102019194245

16. Silva RCD, Rosa ACA, Fiuza NO, Machado LCS, Lovatto V, Veneziano LSN, et al. Efeitos da fisioterapia respiratória no pós-operatório de cirurgia bariátrica. RECIFAQUI [Internet]. 2020;1(10):1-23. Disponível em: http://recifaqui.faqui.edu.br/ index.php/recifaqui/article/view/7

17. Stefanello RB, Prazeres SM, Santos FS, Mancia JR, Leal SMC. Caracterização de pacientes com lesões de pele hospitalizados em unidade de internação clínico-cirúrgica. Enferm Foco [Internet]. 2020;11(2):105-11. Disponível em: http://revista.cofen.gov.br/ index.php/enfermagem/article/view/3294/796
18. Barbosa TP, Beccaria LM, Poletti NAA. Avaliação do risco de úlcera por pressão em UTI e assistência preventiva de enfermagem. Revista Enfermagem UERJ [Internet]. 2014;22(3):353-8. Disponível em: https://www.e-publicacoes.uerj. br/index.php/enfermagemuerj/article/view/13724

19. Moro JV, Caliri MHL. Úlcera por pressão após a alta hospitalar e o cuidado em domicílio. Esc. Anna Nery [Internet]. 2016;20(3):e20160058. Disponível em: http://www.scielo.br/scielo. php?script=sci arttext\&pid=S1414-81452016000300203\&lng=pt

20. Mendonça PK, Loureiro MDR, Frota OP, Souza AS. Prevenção de lesão por pressão: ações prescritas por enfermeiros de centros de terapia intensiva. Texto contexto - enferm. 2018;27(4):e4610017. http://dx.doi.org/10.1590/0104$\underline{07072018004610017}$

21. Maciel DO, Freitas KO, Santos BR, Torres RSC, Reis DST, Vasconselos EV. Percepções de pacientes adultos sobre a unidade de terapia intensiva. Enferm Foco [Internet]. 2020;11(1):14752. Disponível em: http://revista.cofen.gov.br/index.php/ enfermagem/article/view/2071/720

22. Mercês MO, Carvalho BF, Silva DR, Passos SC. O acolhimento da família na unidade de terapia intensiva. Rev Enf Contemp. 2019;8(2):182-9. https://doi.org/10.17267/2317-3378rec.v8i2.2534 